\title{
Design Of Wireless Sensor Networks For IOT Application : A Challenges and survey
}

\author{
Mr.K.Muruganandam, Dr.B.Balamurugan, Dr.Sibaram Khara \\ Asst.Professor, School.of. SEECE, Gagotias University,Greater Noida. \\ Professor, School.of.SECE, Galgotias University, Greater Noida. \\ Dean, School.of.SEECE, Galgotias University, Greater Noida.
}

\begin{abstract}
:
Internet of things (IOT) Can be defined in many ways it encompasses many aspects of life such as connected homes, connected cities, connected cars and roads, roads to devices that track an individual's behaviour. It is expected that one trillion Internet-connected devices will be available with mobile phones as the eyes and ears of the applications connecting all of those connected things. IoT made it possible for billions objects to communicate over worldwide over a public, private internet protocol network In 2010. In 2010 -11 the number of everyday physical objects and devices connected to the Internet was around 12.5 billion. The essential idea of the Internet of Things (IoT) has been around for nearly two decades, and has attracted many researchers and industries because of its great estimated impact in improving our daily lives and society. When things like household appliances are connected to a network, they can work together in cooperation to provide the ideal service as a whole, not as a collection of independently working devices. This is useful for many of the real-world applications and services, and one would for example apply it to build a smart residence; windows can be closed automatically when the air conditioner is turned on, or can be opened for oxygen when the gas oven is turned on. The idea of IoT is especially valuable or persons with disabilities, as IoT technologies can support human activities at larger scale like building or society, as the devices can mutually cooperate to act as a total system. WSNs are integrated into the "Internet of Things", where sensor nodes join the Internet dynamically, and use it to collaborate and accomplish their tasks. Wireless sensor networks (WSN) are well suited for long-term environmental data acquisition for IoT representation. In this paper, it is proposed to implement a WSN platform that can be used for a range of long-term environmental monitoring for IoT applications. This paper presents functional design of WSN for IoT application. To provide a comprehensive overview of the IoT scenario and reviews its enabling technologies and the sensor networks. Also, it describes a six-layered architecture of IoT and points out the related key challenges.
\end{abstract}

Key words : IoT, Sensor Node ,Energy Efficiency, Wireless Sensor Networks, privacy, security, IoT Applications.

\section{Introduction :}

The Internet of Things (IoT) is the interconnection of uniquely identifiable embedded computing devices within the existing Internet framework. Typically, IoT is expected to offer advanced connectivity of devices and systems, and services that goes beyond M2M i.e. machine-to- machine(M2M) communications and covers a variety of protocols, various domains, and applications. The interconnection of all these embedded devices which also includes smart objects, is expected to lead in automation in nearly all fields enabling advanced applications like a Smart Grid. Objects or things communicate with each other and perform the required actions. Human does not need to interact with system. IoT system is made up of three components: sensor, actuator, connectivity devices. Despite these important benefits, there was broad agreement among participants that increased connectivity between devices and the Internet may create a number of security and privacy risks. According to panelists, IoT devices may present a variety of potential security risks that could be exploited to harm consumers by: (1) enabling unauthorized access and misuse of personal information; (2) facilitating attacks on other systems; and (3) creating safety risks. Although each of these risks exists with traditional computers and computer networks, they are heightened in the IoT, as explained further below.First, on IoT devices, as with desktop or laptop computers, a lack of security could enable intruders to access and misuse personal information collected and transmitted to or from the device. For example, new smart televisions enable consumers to surf the Internet, make purchases, and share photos, similar to a laptop or desktop computer. Like a computer, any security vulnerabilities in these televisions could put the information stored on or transmitted through 
the television at risk. If smart televisions or other devices store sensitive financial account information, passwords, and other types of information, unauthorized persons could exploit vulnerabilities to facilitate identity theft or fraud. Thus, as consumers install more smart devices in their homes, they may increase the number of vulnerabilities an intruder could use to compromise personal information.

The basic idea of IoT is to allow autonomous exchange of useful information between invisibly embedded different uniquely identifiable real world devices around us, fueled by the leading technologies like Radio-Frequency IDentification (RFID) and Wireless Sensor Networks (WSNs) which are sensed by the sensor devices and further processed for decision making, on the basis of which an automated action is performed.

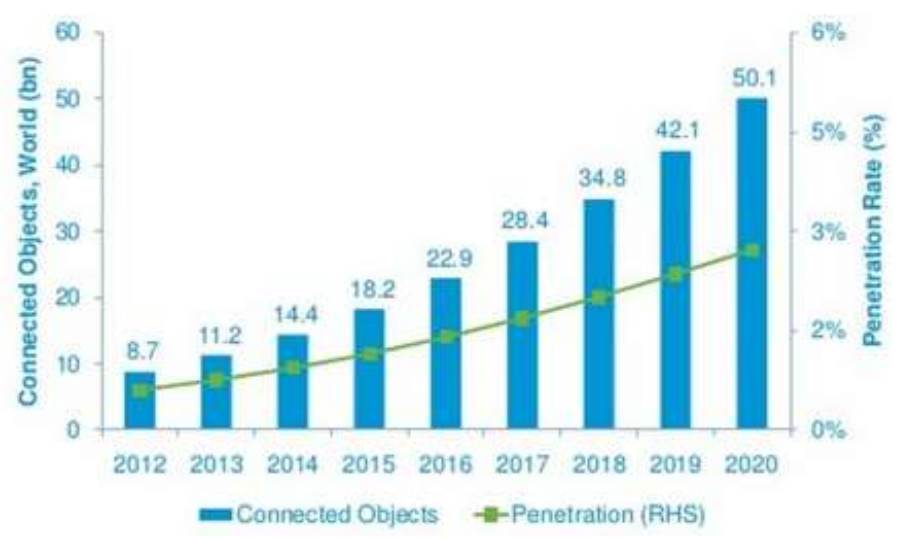

Fig. 1. Expected penetration of connected objects by the year 2020, according to focus on Survey.

\section{Energy Efficiency:}

We need to create a set of task and find out each task which sensor is required, and for executing this task we will turn on sensor for particular time interval and after completion of task sensor will go to idle state. So, in this way we are trying to improve energy efficiency of system. This is called as duty cycling. Efficient heterogeneous sensing of the urban environment needs to simultaneously meet competing demands of multiple sensing modalities. This has implications on network traffic, data storage, and energy utilization. Importantly, this encompasses both fixed and mobile sensing infrastructure as well as continuous and random sampling. A generalized framework is required for data collection and modelling that effectively exploits spatial and temporal characteristics of the data, both in the sensing domain as well as the associated transform domains.

\section{Definitions:}

There is no unique definition available for Internet of Things that is acceptable by the world community of users. In fact, there are many different groups including academicians, researchers, practitioners, innovators, developers and corporate people that have defined the term. The best definition for the Internet of Things would be: "An open and comprehensive network of intelligent objects that have the capacity to auto-organize, share information, data and resources, reacting and acting in face of situations and changes in the environment". Internet of Things is maturing and continues to be the latest, most hyped concept in the IT world. Over the last decade the term Internet of Things (IoT) has attracted attention by projecting the vision of a global infrastructure of networked physical objects, enabling anytime, anyplace connectivity for anything and not only for any one. The Internet of Things can also be considered as a global network which allows the communication between human-to-human, human-to-things and things-to-things, which is anything in the world by providing unique identity to each and every object. IoT describes a world where just about anything can be connected and communicates in an intelligent fashion that ever before. Most of us think about "being connected" in terms of electronic devices such as servers, computers, tablets, telephones and smart phones. In what's called the Internet of Things, sensors and actuators embedded in physical objects from roadways to pacemakers are linked through wired and wireless networks, often using the same Internet IP that connects the Internet. These networks churn out huge volumes of data that flow to computers for analysis.

\section{IOT Vision:}

In 2005, ITU reported about a ubiquitous networking era in which all the networks are interconnected and everything from tires to attires will be a part of this huge network. Imagine yourself doing an internet search for your watch you lost somewhere in your house. So this is the main vision of IoT, an environment where things are able to talk and their data can be processed to perform desired tasks through machine learning . A practical implementation of IoT is demonstrated by a soon-to-be released Twine, a compact and low-power hardware working together with real-time 
web software to make this vision a reality. However different people and organizations have their own different visions for the IoT .

\section{IOT Architecture :}

For further development of IoT, a number of multi-layered security architectures are proposed. It is described a three key level architecture of IoT while described a four key level architecture. proposed a five layered architecture using the best features of the architectures of Internet and Telecommunication management networks based on TCP/IP and TMN models respectively. Similarly a six-layered architecture was also proposed based on the network hierarchical structure . So generally it's divided into six layers as shown in the below Fig..

The six layers of IoT are described below:

\section{Coding Layer}

Coding layer is the foundation of IoT which provides identification to the objects of interest. In this layer, each object is assigned a unique ID which makes it easy to discern the objects.

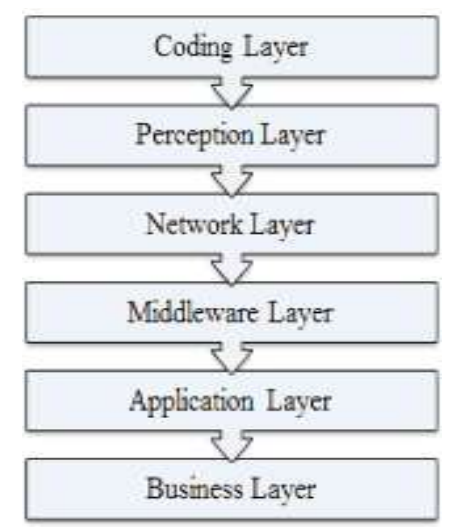

\section{Perception Layer}

This is the device layer of IoT which gives a physical meaning to each object. It consists of data sensors in different forms like RFID tags, IR sensors or other sensor networks which could sense the temperature, humidity, speed and location etc of the objects.This layer gathers the useful information of the objects from the sensor devices linked with them and converts the information into digital signals which is then passed onto the Network Layer for further action.

\section{Network Layer}

The purpose of this layer is receive the useful information in the form of digital signals from the Perception Layer and transmit it to the processing systems in the Middleware Layer through the transmission mediums likeWiFi, Bluetooth,WiMaX, Zigbee, GSM, 3G,etc with protocols like IPv4, IPv6, MQTT, DDS etc.

\section{Middleware Layer}

This layer processes the information received from the sensor devices . It includes the technologies like Cloud computing, Ubiquitous computing which ensures a direct access to the database to store all the necessary information in it. Using some Intelligent Processing Equipment, the information is processed and a fully automated action is taken based on the processed results of the information.

\section{Application Layer}

This layer realizes the applications of IoT for all kinds of industry, based on the processed data. Because applications promote the development of IoT so this layer is very helpful in the large scale development of IoT network. The IoT related applications could be smart homes, smart transportation, smart planet etc.

\section{Business Layer}

This layer manages the applications and services of IoT and is responsible for all the research related to IoT. It generates different business models for effective business strategies .

\section{Wireless Sensor Networks}

Technology advances in wireless communications and electronics have enabled the development of low-cost, lowpower, multi-functional sensor nodes that are small in size and communicate untethered in short distances. These tiny and generally simple sensor nodes consist of sensing units, data processing, and communicating components . A large 
number of such nodes deployed over large areas can collaborate with each other. To be cost-effective, the sensor nodes often operate on very restricted energy reserves. Premature energy depletion can severely limit the network service and needs to be addressed considering the IoT application requirements for cost, deployment, maintenance, and service availability. Open nature deployments and communication protocol developments and experiments show that WSN optimization for reliable operation is time-consuming and costly. It hardly satisfies the IoT applications requirements for long-term, low-cost and reliable service, unless reusable hardware and software platforms are available, including flexible Internet enabled servers to collect and process the field data for IoT applications.

This paper contributions of interest for researchers in the WSN field can be summarized as: 1) detailed specifications for a demanding WSN application for long-term environmental monitoring that can be used to analyze the optimality of novel WSN solutions, 2) specifications, design considerations, and experimental results for platform components that suit the typical IoT application requirements of low cost, high reliability, and long service time, 3) specifications and design considerations for platform re-usability for a wide range of distributed event-based environmental monitoring applications, and 4) a fast and configuration-free field deployment procedure suitable for large scale IoT application deployments.

\section{Applications of WSN}

WSN is a bi-directional wirelessly connected network of sensors in a multi-hop fashion, built from several nodes scattered in a sensor field each connected to one or several sensors which can collect the object specific data such as temperature, humidity, speed etc and then pass on to the processing equipment . The sensing nodes communicate in multi-hop Each sensor is a transceiver having an antenna, a micro-controller and an interfacing circuit for the sensors as a communication, actuation and sensing unit respectively along with a source of power which could be both battery or any energy harvesting technology . However has proposed an additional unit for saving the data, named as Memory Unit which could also be a part of the sensing node. A typical sensing node is shown in the figure below:

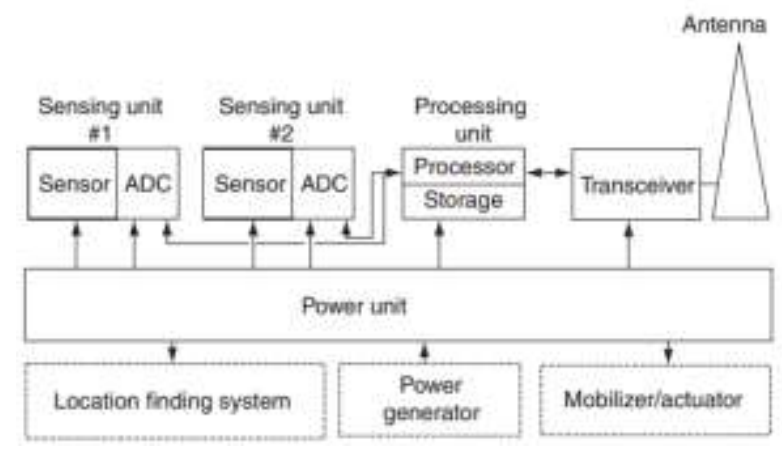

Fig. 4. A typical sensing node

Wireless Sensors Network technology and RFID technology when combined together opens up possibilities for even more smart devices, for which a number of solutions have been proposed . An example solution is provided by the Intel Research Labs in the form of Wireless Identification Sensing Platform (WISP). WISP is a passive wireless sensor network with built-in light, temperature and many other sensors .Both WSN and RFID Sensor Networks have their own advantages but RFID Sensor Networks have a low range and their communication is Asymmetric while WSNs have a comparatively longer range and their communication is Peer-to-Peer. Moreover most of the WSNs are based on the IEEE 802.15.4 standard, which specifies the Physical and MAC layer of Low-Rate Wireless Personal Area Networks (LR-WPANs). The technologies that enables the integration of WSN with the IOT are a hot research topic, many solutions have been proposed for that including that of a 6LOWPAN standard , that allows IPv6 packets to be transmitted through the networks that are computationally restricted. Also there's ROLL routing standard for end-to-end routing solutions.

Our purpose is to minimize energy consume by sensor and improve its efficiency. Sensor collects the huge amount of data from environment which we are storing in database user always looking for short and important data or information from database so our purpose is to fulfil user's expectations by using data mining algorithm for accessing data from database. Data mining is technique used to extract short and important data from enormous amount of data. Keeping the sensor always in active state required large amount of energy so to reduce this energy consumption we will switch sensor from active to idle and idle to active state as per user's request.. System should take decisions from its past experiences. That is system should behave rationally. Sensor daily collects the data and stores it in the cloud. Cloud is accessible only to the authorized user. Someone should not alter or change the data in the cloud. We are going to use strong authentication technique for this purpose. 


\section{Future Scope And Applications :}

Most of the daily life applications that we normally see are already smart but they are unable to communicate with each other and enabling them to communicate with each other and share useful

information with each other will create a wide range of innovative applications .

Smart Home: IoT will also provide DIY solutions for Home Automation with which we will be able to remotely control our appliances as per our needs. Proper monitoring of utility meters, energy and water supply will help saving resources and detecting unexpected overloading, water leaks etc. There will be proper encroachment detection system which will prevent burglaries.

Smart Gardening : Gardening sensors will be able to measure the light, humidity, temperature, moisture and other gardening vitals, as well as it will water the plants according to their needs.

Smart Environment: Prediction of natural disasters such as flood, fire, earthquakes etc will be possible due to innovative technologies of IoT. There will be a proper monitoring of air pollution in the environment.

Smart Hospitals: Hospitals will be equipped with smart flexible wearable embedded with RFID tags which will be given to the patients on arrivals, through which not just doctors but nurses will also be able to monitor heart rate, blood pressure, temperature and other conditions of patients inside or outside the premises of hospital . There are many medical emergencies such as cardiac arrest but ambulances take some time to reach patient, Drone Ambulances are already in the market which can fly to the scene with the emergency kit so due to proper monitoring, doctors will be able to track the patients and can send in the drone to provide quick medical care until the ambulance arrive.

\section{Smart Transportation}

The development of smart transportation is generally led by governments or transportation authorities. Successful examples include real time traffic and public transportation information sharing, intelligent traffic control systems, incentive program to regulate transportation, largely promotion of electric vehicle and charging facilities, and dedicated short-range communication (DSRC) enabled vehicular communication system,

a) Navigation and Safety: Utilizing the vehicles (e.g., cars, buses, trains) along with the roads and the rails equipped with sensors, actuators and processing power, important traffic information could be offered to the drivers or passengers of the vehicles to achieve better navigation and safety.

b) Road Planning and Route Optimization: Benefiting from the more accurate traffic information about road patterns, governmental authorities could better plan and design the roads. Particularly, intelligent roads can be performed, with warning messages based on climate conditions and unexpected events (e.g., accidents or traffic jams).

\section{Real Time Management:}

It is a challenging issue for resource constrained sensor networks. In this case, the IoT system needs to rely on efficient service gateway design to minimize the amount of data to be sent by constantly reviewing the data from users, and intelligent data oriented middleware design to only transmit real time information when a reading is out-ofthreshold.

\section{Security and Privacy:}

Security, trust and privacy are also important issues to be considered in practical applications. There are both hard way and soft way methods to achieve different degrees of security. These security methods are appropriate for M2M deployments where there is an existing trust relationship between the devices and server.

\section{Conclusions :}

There is innumerable usefulness of IoT applications into all the domains including medical, manufacturing, industrial, transportation, education, governance, mining, habitat etc. Though IoT has abundant benefits, there are some flaws in the IoT governance and implementation level. The key observations in the literature are that (1) There is no standard definition in worldwide (2) Universal standardizations are required in architectural level (3) Technologies are varying from vendor-vendor, so needs to be interoperable (4) For better global governance, we need to build standard protocols. All the system in existing mechanism implements either energy efficiency or providing quality of information. System which provides quality of information needs more energy for good performance. It is possible to implement data mining technique and machine learning algorithm for quality of performance of system with efficient energy consumption.

\section{References :}

[1] J. A. Stankovic, "Research directions for the Internet of Things," IEEE Internet Things J., vol. 1, no. 1, pp. 3-9, Feb. 2014.

[2] Zhengguo Sheng, Chinmaya Mahapatra Chunsheng Zhu And Victor C. M. Leung, "recent advances in industrial wireless Sensor networks toward efficient management in IOT", volume 3, 2015. 
[3] Huansheng Ning, Hong Liu and Laurence T. Yang "Aggregated- Proof Based Hierarchical Authentication Scheme for the Internet of Things", VOL. 26, NO. 3, MARCH 2015

[4] V. G. Tharinda Nishantha Vidanagama, Daisuke Arai, And Tomohiko Ogishi, "service environment for smart wireless devices:an m2m gateway selection scheme", volume 3, 2015.

[5] Katsuya Sutox, Hiroki Nishiyamax, NeiKatox, Chih-Wei Huang," an energy-efficient and delay- aware wireless computing system for industrial wireless sensor networks", 2169-3536 (c) 2015

[6] Jin Qi1, Xiaoxuan Hu, Yun Ma1, And Yanfei Sun “, a hybrid security and compressive sensing- based sensor data gathering scheme", volume 3, 2015.

[7] Perera, Member, C.Harold Liu \& S.Jayawardena - The Emerging Internet of Things Marketplace From an Industrial Perspective: A Survey

[8] A. Zanella,N. Bui, A. Castellani \& L. Vangelista, M.e Zorzi, Internet of Things for Smart Cities IEEE INTERNET OF THINGS JOURNAL, VOL. 1, NO. 1, FEBRUARY 2014

[9] S. Rajguru, S. Kinhekar \& S. Pati ,Analysis of Internet of Things in a Smart Environment Vol. 4 Issue 4, April-2015, pp: (40-43)

[10]Air Quality Egg, —Air Quality Egg,\| 2013. Retrieved From http://airqualityegg.com/

[11] Amrita Vishwa Vidya Peetham, -Amritawna: Amrita center for wireless networks and applications, $\| 2013$.

[12]Menon1, et al. " Implementation of internet of things in bus transport system of singapore"Asian Journal of Engineering Research(2013).

[13]Shao-Lei Zhai et.al " Research of Communication Technology on IOT for High-Voltage Transmission Line " International Journal of Smart Grid and Clean Energy(2012)

[14]S.Pandikumar, R.S. Vetrivel, "Internet of Things Based Architecture of Web and Smart Home Interface Using GSM",International Journal of Innovative Research in Science, Engineering and Technology Volume 3, Special Issue 3, March 2014 